\title{
Moderate Nutritional Risk among Community-Dwelling Canadian Older Men in the Manitoba Follow-up Study (MFUS)
}

CO Lengyel ${ }^{1}$, CT Moodoo ${ }^{1}$, RB Tate ${ }^{2}$. ${ }^{1}$ Department of Food and Human Nutritional Sciences, Faculty of Agricultural \& Food Sciences;

${ }^{2}$ Department of Community Health Sciences, Max Rady College of Medicine,

University of Manitoba, Winnipeg, MB

\section{INTRODUCTION}

(www.mfus.ca)

The first step in identifying nutrition risk in older adults is through nutrition screening.

Nutrition screening can be defined as "a process to identify an individual who is malnourished or who is at risk for malnutrition to determine if a detailed nutrition assessment is indicated" (Mueller, 2011).

There are differing protocols set in place for community nutrition screening tools most commonly used in nutrition practice and for those who score as either low, medium and high nutrition risk.

Trajectory analysis is used in longitudinal studies to subdivide the research sample into distinct groups to identify clusters of individuals who encompass similar trajectories overtime.

Five nutritional risk trajectory categories were identified for the MFUS participants (all males): 1) Low (L); 2) Moderate (M);3) Moderate-Increase (MI); 4) High (H) and 5) High-Increase (HI) (Lengyel et al., 2017)

The protocol for those who score as moderate nutritional risk is often times less aggressive than the protocol for those who screen as high nutritional risk Most often, individuals who score as moderate nutritional risk are placed lower on the priority list; often they get left alone or reassessed less frequently than individuals who score as high nutritional risk.

Based on the Lengyel et al. (2017) results, this study will take a closer look at the older adult men who participated in the MFUS in 2007 and scored as moderate nutritional risk but then fall into two out of the five trajectory groups identified:

moderate risk (M) and;

moderate-increase risk (MI)

\section{OBJECTIVES}

To examine characteristic differences between MFUS participants at 2007 to predict trajectory group classification

To compare the 16-nutrition risk (NR) items on SCREEN II among the two groups at 2007 to identify specific NR items that better predict trajectory group allocation

To determine NR items that showed the biggest decline over a 4-year period among those in the Ml trajectory group

\section{METHODOLOGY}

SCREEN II Survey

SCREEN II (Seniors in the Community: Risk Evaluation for Eating and Nutrition) is a validated nutritional risk screening tool for community-living older adults (Keller, Goy \& Kane, 2005) Nutritional Risk (NR) scores were calculated from 16 components

Assessing Nutritional Risk in MFUS Cohort:

Five SCREEN II surveys from 2007-2011

In 2011, 336 men

Age of respondents (Mean $\pm \mathrm{SD}$ ):

90.2 years \pm 3.1

117 men in the M Trajectory Group

Data Analysis

49 men in the MI Trajectory

Trajectories of nutritional risk scores: semi-parametric groupbased trajectory approach

Descriptive characteristics: t-tests and $x^{2}$

$M$ and MI NR items comparison in 2007: $x^{2} p<0.05$

NR items that decline from 2007-2011 amongst MI trajectory group: $x^{2}$

\section{Manitoba Follow-up Study (MFUS)}

ongest-running prospective longitudinal study of cardiovascular disease in Canada

Cohort sealed on July 1, 1948 with 3,983 men

- Royal Canadian Air Force aircrew recruits

- Mean age at baseline $31 y$, with $90 \%$ of cohort $20-39 y$ of age

Primary objective has been to monitor morbidity and mortality of cardiovascular disease

During the 1990s the study's focus on cardiovascular epidemiology shifted to examining issues of aging

- Successful Aging Questionnaires (SAQ) administered in 1996, 2000, 2002, and annually 2004-2015

After 70 years of follow-up, 180 men were alive, at a mean age of 96 years (July 1, 2017)

This study has approval from the University of Manitoba Health Research Ethics Board

Thanks to the members of the Manitoba Follow-up Study for their continuous participation and enthusiasm.

The research team acknowledges funding from the Canadian Institutes of Health Research, the Manitoba Health Research Council, the University of Manitoba Centre on Aging and the Dr. Paul H.T. Thorlakson Foundation Fund.

\section{RESULTS}

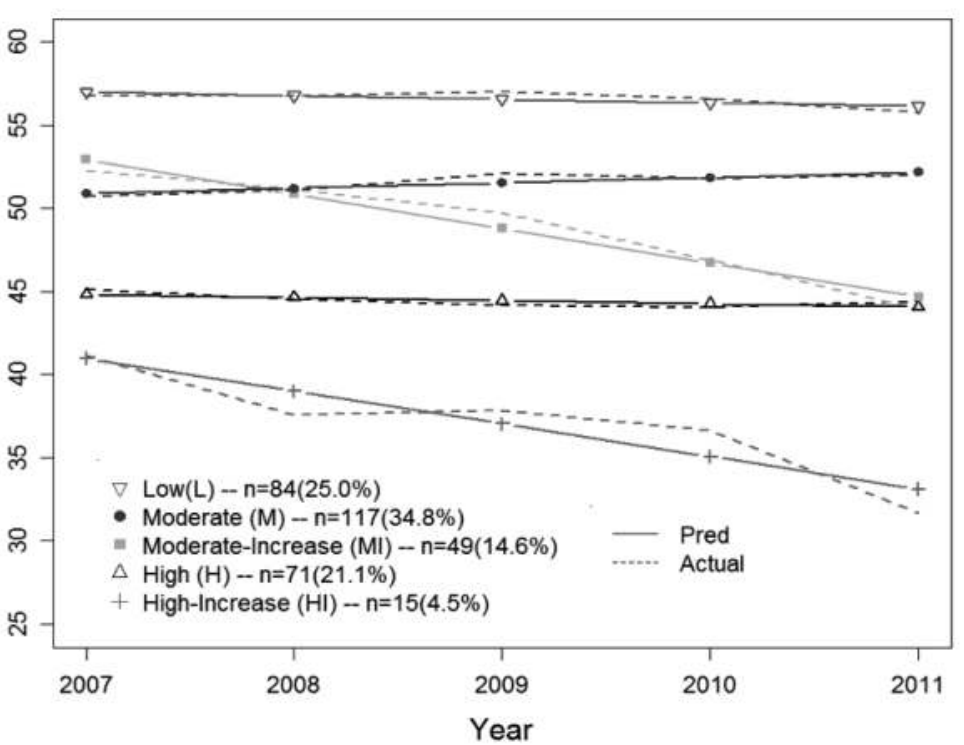

Figure 1. Trajectories of Nutritional Risk among very Old Community-Dwelling Men

Table 1. Descriptive Characteristics in 2007 of The MFUS Participants by Nutritional Risk Trajectory Group $(n=166)$

\begin{tabular}{|c|c|c|c|}
\hline & \multicolumn{2}{|c|}{ Nutrition Risk Trajectories } & \multirow[b]{2}{*}{$\mathrm{p}$-value } \\
\hline & Moderate (M) & $\begin{array}{l}\text { Moderate- } \\
\text { Increase (MI) }\end{array}$ & \\
\hline$\overline{\mathrm{Age}, \text { mean (SD) })^{2}}$ & $\begin{array}{l}n=117 \\
86.0(3.2)\end{array}$ & $\begin{array}{l}n=49 \\
86.2(2.8)\end{array}$ & 0.74 \\
\hline SF-36 MCS, mean (SD) & $55.8(7.5)$ & $55.5(7.2)$ & 0.86 \\
\hline SF-36 PCS, mean (SD) & $42.9(9.9)$ & $42.8(9.1)$ & 0.91 \\
\hline BMI, mean (SD) & $25.1(2.9)$ & $25.2(3.1)$ & 0.79 \\
\hline Live alone, $\mathbf{n}(\%)$ & $17 \%$ & $22 \%$ & 0.41 \\
\hline Married & $79 \%$ & $71 \%$ & 0.32 \\
\hline \multicolumn{4}{|l|}{$\begin{array}{l}\text { Pre-existing health } \\
\text { conditions: }\end{array}$} \\
\hline $\begin{array}{l}\text { Oonomis } \\
\text { Ischemic Heart Disease }\end{array}$ & $16.2 \%$ & $24.4 \%$ & 0.21 \\
\hline Stroke & $6.0 \%$ & $6.1 \%$ & 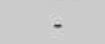 \\
\hline Diabetes & $6.8 \%$ & $4.1 \%$ & \\
\hline C.O.P.D. & $1.0 \%$ & $2.0 \%$ & - \\
\hline Peripheral Artery Disease & $6.0 \%$ & $4.1 \%$ & \\
\hline Cancer & $8.6 \%$ & $14.3 \%$ & 0.26 \\
\hline $\begin{array}{l}\text { Self-rating on how healthy } \\
\text { their diets are }\end{array}$ & $75.5(19.2)$ & $78.6(19.8)$ & 0.38 \\
\hline $\begin{array}{l}\text { Self-rating of the importance } \\
\text { Nutrition has on healthy } \\
\text { aging }\end{array}$ & $83.1(18.6)$ & $85.6(13.4)$ & 0.40 \\
\hline Aged successfully, n(\%) & $84.6 \%$ & $81.6 \%$ & 0.63 \\
\hline
\end{tabular}

Table 2. Participants in the Moderate and ModerateIncrease Trajectory Groups Scoring in the 'Best' Category on each of the SCREEN II Items in 2007

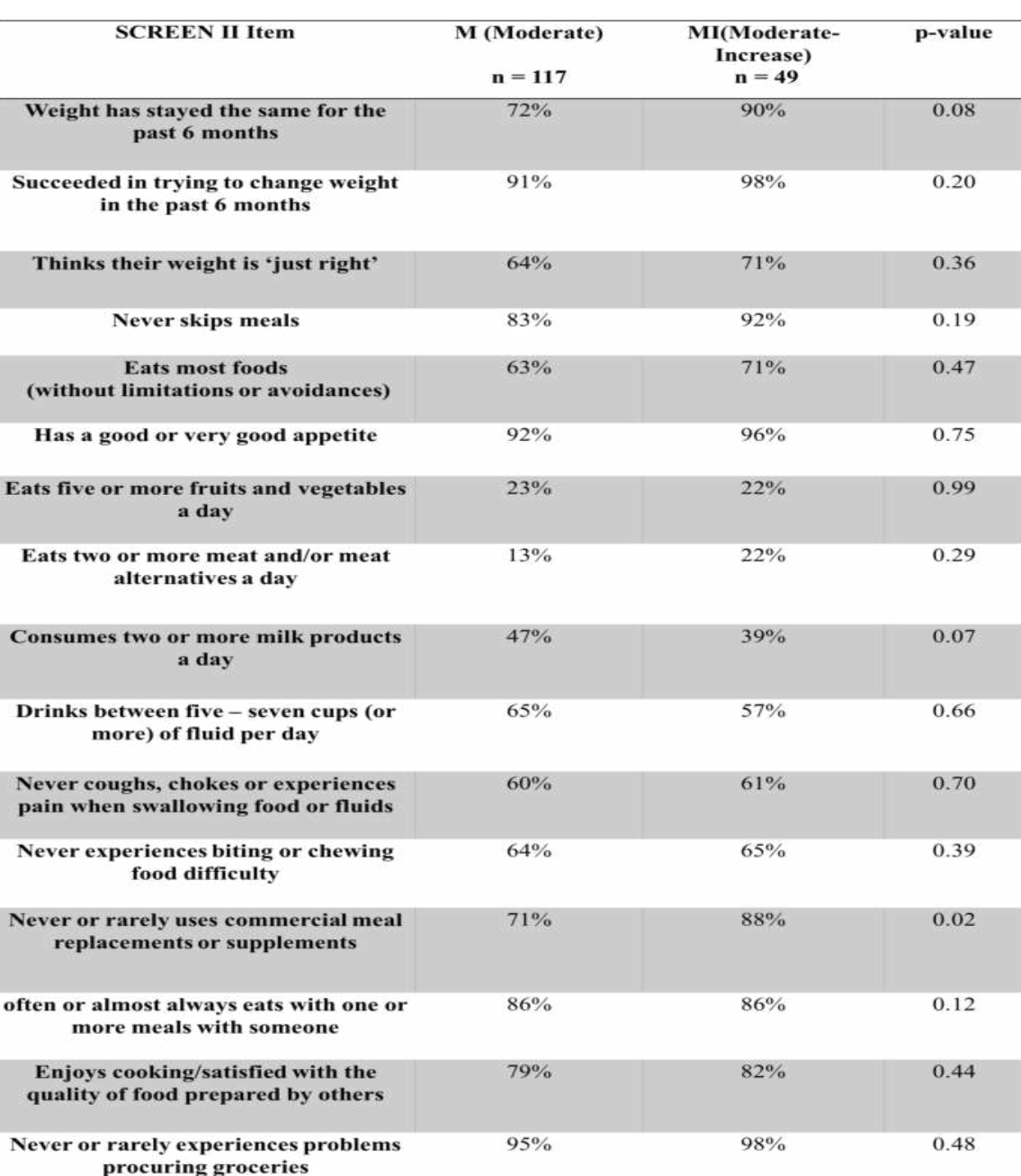

Table 3. Participants in the Moderate-Increase Trajectory Group with a Decline in Their Response from 2007 and Each Year After

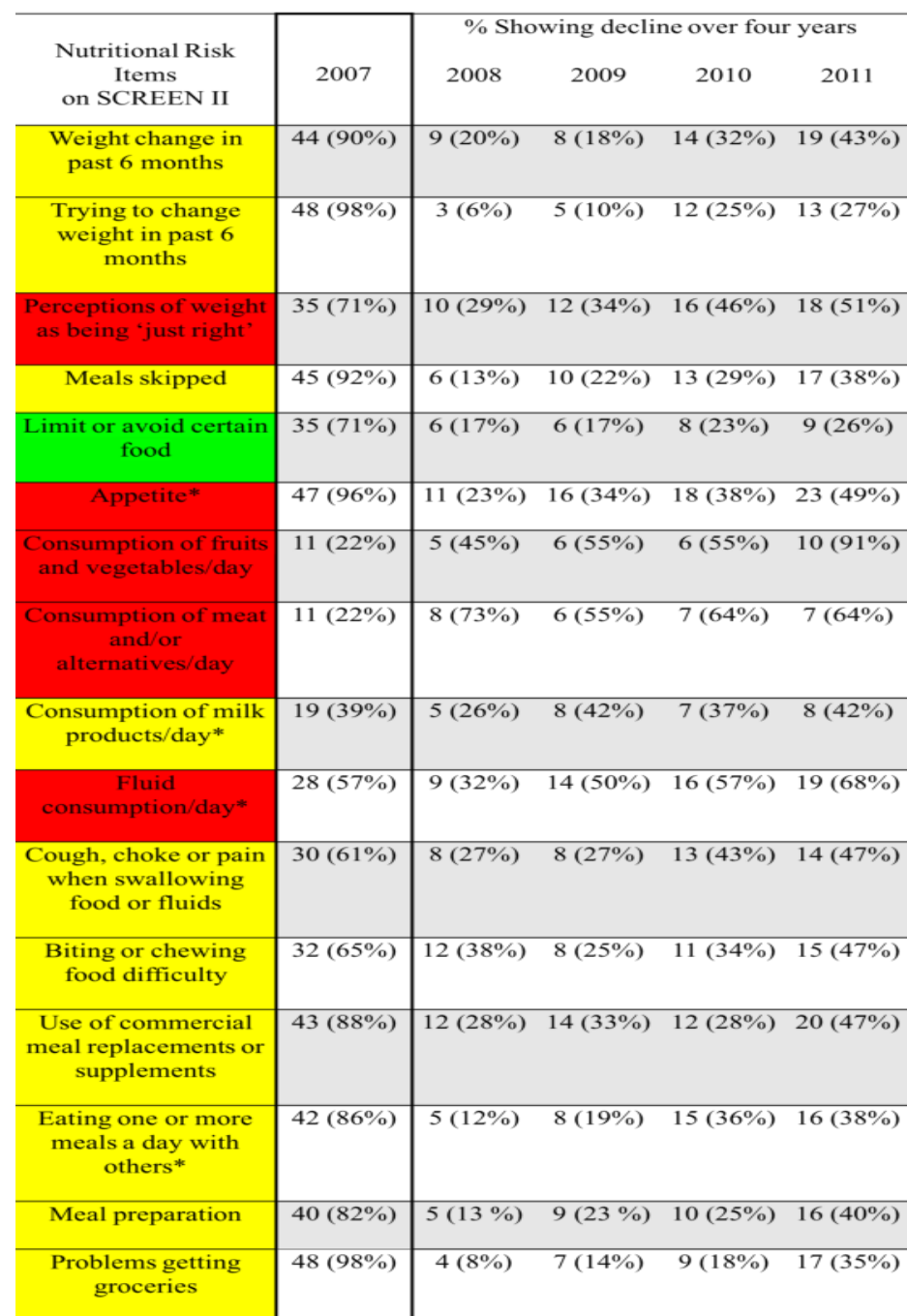

Individuals who screen as moderate nutritional risk are at an increased risk for nutritional decline over a four-year period

Individuals who screened as moderate nutritional risk should be reassessed as often as individuals who fall into the high nutrition risk category

The SCREEN II items: perceptions of weight as being just right, appetite, fruit and vegetable consumption and meat/alternative per day along with daily fluid intake, showed more than $50 \%$ decline over a four-year period. These items should be monitored more closely as indicators for possible decline in nutrition risk especially for men 